\title{
The Work of David Gottlieb: A Success Story
}

\author{
Bertil Gustafsson* \\ Division of Scientific Computing, Uppsala University, Box 337, \\ SE-75105 Uppsala, Sweden.
}

Received 1 January 2010; Accepted (in revised version) 1 March 2010

Available online 17 September 2010

To the memory of David Gottlieb

\begin{abstract}
This article is a brief survey of David Gottlieb's extraordinary research career. It is impossible to give a thorough presentation of all his research and the impact of his work, but we shall describe the main contributions and give examples of the results in some of his papers. David was for many years the dominating person in the development of spectral methods, and we devote much of the space in this article to this field.
\end{abstract}

\section{Contents}

1 Introduction 481

2 Life in two countries $\quad 482$

3 Spectral methods 483

4 Gibbs phenomenon $\quad 487$

5 High order difference methods 489

6 Open boundaries and the PML method 491

7 Other areas 492

8 Conclusions 493

\section{Introduction}

This article is a brief survey of David Gottlieb's extraordinary research career. He published 125 papers and two books, and made an outstanding contribution to the field of numerical analysis. He had an unusual width in his work, and most types of numerical methods for PDE are included in his work. Fig. 1 shows an approximate distribution of his interest and production over the years.

*Corresponding author. Email address: bertil.gustafsson@it.uu.se (B. Gustafsson) 


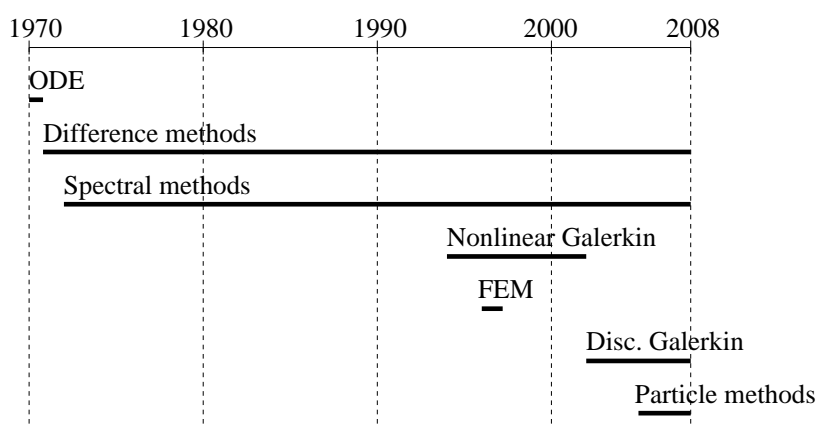

Figure 1: Distribution of David Gottlieb's research.

The first part containing some theoretical results about ordinary differential equations originates from his Master's work at Tel Aviv University.

It is impossible to give a thorough presentation of all his research and the impact of his work, but we shall describe the main contributions and give examples of the results in some of his papers. David was for many years the dominating person in the development of spectral methods, and we devote much of the space in this article to this field.

The list of publications at the end includes slightly more than a third of David's papers, and is limited to references made in the text. There are certainly many other significant papers, and many other coauthors who should be mentioned. But this would require a more complete biography.

\section{Life in two countries}

Born in Tel Aviv 1944, David Gottlieb spent his first 28 years in Israel. Like everyone else he had to make his military service, which lasted for three years. But not only that. He hade to fight a war in 1967, a kind of experience that is not shared by many mathematicians in the world.

Even if he was a talented mathematician, his main interest in early life was history. When he was wandering around the campus of Tel Aviv University to look for the history department, he encountered a mathematics professor who was inquiring about his errand. After a short conversation, David was invited to the mathematics department, and that was the start of his academic career in mathematics. The Masters degree under S. Breuer was followed by the PhD 1972 with Saul Abarbanel as the advisor.

Abarbanel had spent many years at MIT in Boston, and he arranged for David to visit the Department of Applied Mathematics there as a postdoc. His thesis was about construction of difference methods of split type for nonlinear partial differential equations, and it was therefore quite natural to work with one of the world's best specialists in that topic, Gilbert Strang. However, things took a different turn when he met Steve Orszag, who was interested in spectral methods for PDE. This came to be David's main research line. 


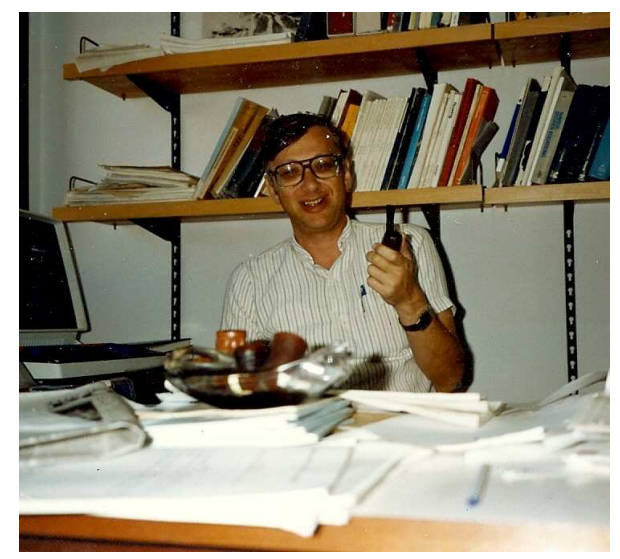

Figure 2: David Gottlieb at his office at Tel Aviv University 1977.

In 1973 the Institute of Computer Applications in Science and Engineering (ICASE) was created, mainly sponsored by NASA and located at NASA Langley Research Center in Hampton, Virginia. ICASE sponsored summer visits by researchers who were interested in applications, mainly fluid dynamics. David made his first visit there 1974, and it was followed by frequent visits until the end of ICASE' existence.

In 1976 it was time to decide whether or not to stay in USA. David and his wife had strong ties to Israel, and the decision was to go back. He got a position as Senior Lecturer at the mathematics department of Tel Aviv University, and his career went straight upwards from there. The picture above (Fig. 2) shows him at his office in Tel Aviv 1977.

His ties with USA remained, and every summer he visited ICASE. In 1985 he accepted an offer from Brown University, and from then on, he and his family lived in Providence, R.I., of course with many visits in Israel.

Fig. 3 is a sketch of David's whereabouts over time.

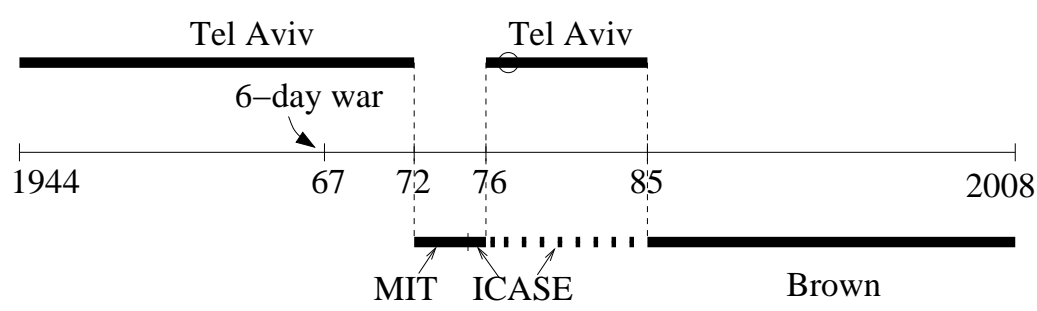

Figure 3: The whereabouts of David Gottlieb.

\section{Spectral methods}

Spectral methods is a very general concept that could apply to all sorts of applications. For a one-dimensional problem the basic idea is to represent the solution $u(x)$ to a certain 
problem as a series

$$
u(x)=\sum_{j} c_{j} \phi_{j}(x)
$$

where the basis functions $\phi_{j}(x)$ are known. The problem is to find the coefficients $c_{j}$. For theoretical purposes the sum may be infinite, but when computing it has to be finite. The most well known basis functions are the Fourier modes $e^{i j x}$, and if the coefficients are found from a given measured function $f(x)$, we talk about spectral analysis. This technique has been known for centuries, but not as a numerical method for computing the solution to PDE. In the late sixties, Steve Orszag was making the first attempts by using Fourier series for problems in turbulence. In 1970 he was visiting National Center for Atmospheric Research (NCAR) in Boulder, Colorado, and made the first large scale computations on the powerful computer that was located there. His first publication was [53] on the Galerkin version. At the same time Heinz Kreiss was visiting the computational group at NCAR with Joe Oliger as one of the members, and they started looking into this new class of methods from a more theoretical point of view. They advocated the collocation spectral method, later called the Pseudospectral method, and the first publication of their results was [51]. It was later followed by [17] written by Kreiss' student Bengt Fornberg.

David Gottlieb came to MIT as a postdoc 1972, and soon he started working with Orszag on spectral methods. They had a broad approach, introducing polynomials as basis functions as well. There are no publications from David on this topic, until the 170 page book [34] appeared 1977. This book came to be the main publication on spectral methods for many years. After this book, David published around 50 papers on the topic. A complete description of the state of the art was given in the new book [49] published 2006 with his daughter Sigal Gottlieb and his coworker Jan Hesthaven at Brown University.

Fig. 4 shows the distribution of his production on spectral methods. The feature with a book at each end is striking.

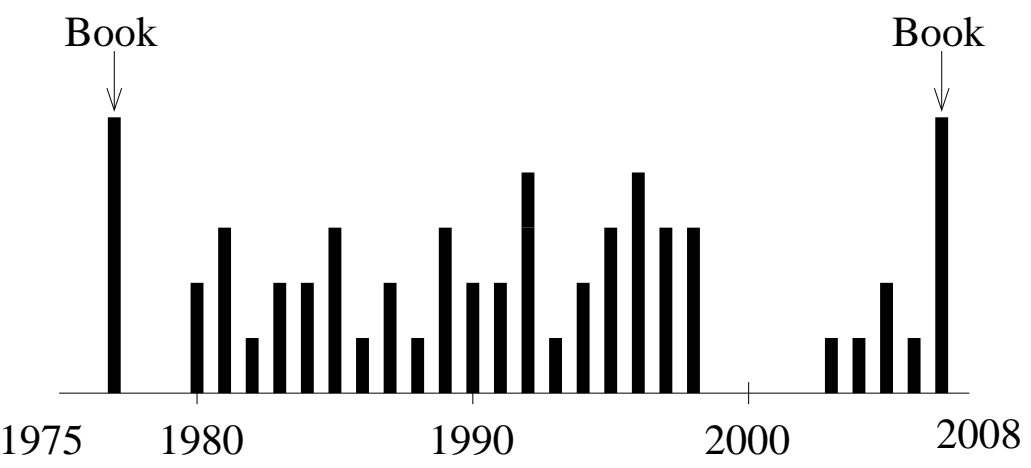

Figure 4: Distribution of publications on spectral methods. 
Even if most of the basic concepts were described in the first book, there were many fundamental difficulties remaining. The major ones were stability, formulation of boundary conditions for polynomial methods, and handling of discontinuities which cause the Gibbs phenomenon.

Beginning with the stability, there were new difficulties already with periodic problems and the Fourier method. The theory for difference methods for this class of problems was fairly complete at this time with the work of Lax and Kreiss as a fundamental part. Fourier methods are of course very well adapted to Fourier analysis leading to the von Neumann condition for constant coefficient equations, but with variable coefficients things become worse. For a hyperbolic PDE

$$
\frac{\partial u}{\partial t}=a(x) \frac{\partial u}{\partial x}
$$

a semidiscrete approximation has the form

$$
\frac{d \mathbf{u}}{d t}=A Q \mathbf{u},
$$

where $\mathbf{u}$ is a vector containing the grid values. The matrix $A$ contain the coefficient values $a\left(x_{j}\right)$ in the diagonal, and the matrix $Q$ has the form $Q=F^{-1} i \omega F$, where $F$ is the discrete Fourier transform. If $Q$ is a difference operator leading to stability for constant coefficients $a(x)=a$, then stability follows for variable Lipschitz continuous coefficients $a(x)$ by the commutation property $Q A(x)=A(x) Q+\mathcal{O}(1)$. Unfortunately this relation does not hold for Fourier methods, and other techniques must be used. There was particular uncertainty about the case where $a(x)$ changes sign in the computational domain. In [35] the issue was settled, and it was shown that the solution satisfies the estimate

$$
\left\|u_{N}(t)\right\| \leq e^{c t}|| u_{N}(0)||+\left|\hat{f}_{N}\right|\left(e^{c t}-1\right),
$$

where $\hat{f}_{N}$ is the highest Fourier coefficient of the initial data. For practical purposes this estimate may not be good enough, since it allows for exponentially growing solutions, and in analogy with difference methods it may be necessary to introduce dissipativity by damping out the higher frequencies.

Compared to difference methods, spectral methods have a more direct connection to the underlying PDE, either by taking scalar products with the differential equation or by requiring that the differential equation is satisfied at the discrete collocation points. These points are carefully chosen for each class of polynomials, and in most cases they are chosen as the so called Gauss-Lobatto points associated with numerical integration. For Chebyshev methods they are $x_{j}=-\cos (j \pi / N)$. The explicit connection between the continuous and discrete case is given by the relation

$$
\sum_{j=0}^{N} \frac{p\left(x_{j}\right)}{\sqrt{1-x_{j}^{2}}} w_{j}=\int_{-1}^{1} \frac{p(x)}{\sqrt{1-x^{2}}} d x, \quad x_{j}=-\cos \left(\frac{j \pi}{N}\right),
$$


which holds for each polynomial $p(x)$ of degree $\leq 2 N-1$ and certain positive weights $w_{j}$. By using this relation, David proved stability in the form

$$
\sum_{j=0}^{N} \frac{u_{N}^{2}\left(x_{j}, t\right)}{\sigma\left(x_{j}\right)} w_{j} \leq \sum_{j=0}^{N} \frac{u_{N}^{2}\left(x_{j}, 0\right)}{\sigma\left(x_{j}\right)} w_{j}
$$

for parabolic equations $u_{t}=\sigma(x) u_{x x}$ with Dirichlet boundary conditions.

A similar relation opened up for a stability proof also for hyperbolic problems of the type

$$
\begin{aligned}
& \frac{\partial u}{\partial t}=a(x) \frac{\partial u}{\partial x}, \quad a(x)>0 \\
& u(1, t)=0 .
\end{aligned}
$$

The results were presented in [23], where also the more difficult problem

$$
\frac{\partial u}{\partial t}= \pm x \frac{\partial u}{\partial x}
$$

was treated.

The time discretization of polynomial spectral methods is a challenge by itself. For Chebyshev polynomials the clustering near the boundaries is obvious from the shape of the cos-function. We have $\cos (\Delta x)=1-\mathcal{O}\left(\Delta x^{2}\right)$, and for hyperbolic equations this leads to severe stability limits of the form $\Delta t \leq \mathcal{O}\left(\Delta x^{2}\right)$ for standard explicit difference approximations. For parabolic equations one gets even more severe conditions of the form $\Delta t \leq \mathcal{O}\left(\Delta x^{4}\right)$. This makes the classical Du-Fort Frankel method an interesting candidate, since it is unconditionally stable for the standard second order difference approximation in space. Actually it was shown in an early paper [25] how to generalize it for the Fourier collocation method. In [31] unconditional stability was proven for the Du-Fort Frankel Chebyshev method.

A new approach was taken in the papers [32,33], where the Kreiss theory based on Laplace transform in time was used. Stability and convergence was proven for systems of hyperbolic initial-boundary value problems.

In [42] stability is proven for Runge-Kutta methods up to order 3 for general Jacobi polynomials including Chebyshev and Legendre polynomials. The article provides a very insightful explanation of how the CFL-limit depends on the eigenvalues of the Sturm-Liouville problem that is associated with the particular type of polynomial. As a consequence, there is a precise stability criterion also for the variable coefficient case. If the variable coefficient is $a(x)$, the criterion has the form

$$
\left|\frac{a\left(x_{j}\right) \Delta t}{\Delta x_{j}}\right| \leq c
$$


for some constant $c$. If the coefficient $a(x)$ is small where the grid points are clustered, this is a less restrictive condition than

$$
\frac{\max _{j}\left|a\left(x_{j}\right)\right| \Delta t}{\min _{j} \Delta x_{j}} \leq c
$$

which is a common type of criterion when standard analysis is used.

The boundary conditions play an important role for the theory as well as for the implementation of polynomial methods. For Galerkin methods, the basis functions are required to satisfy the boundary conditions given for the PDE. This requirement is relaxed in the tau version, such that the combination of all basis functions forming the final numerical solution satisfies the boundary condition, not each basis function by itself.

For the collocation version, this technique may be applied as well, but now with the unknown coefficients determined by the collocation principle. But here is where a new development took place. Penalty methods have been used extensively not only in optimization problems, but also in Galerkin finite element methods. In [18], Daniele Funaro introduced it as a way to enforce the boundary conditions for elliptic problems solved by domain decomposition methods. This idea was now carried over to time dependent hyperbolic problems in joint work with David, see $[19,20]$. The boundary point is included as a collocation point, but an extra penalty term is added to the differential equation. For the scalar case

$$
\begin{aligned}
& \frac{\partial u}{\partial t}=\frac{\partial u}{\partial x}, \quad-1 \leq x \leq 1, \\
& u(1, t)=g(t)
\end{aligned}
$$

and the polynomial approximation $u_{N}(x, t)$, we get the boundary equation

$$
\frac{\partial u_{N}}{\partial t}(1, t)-\frac{\partial u_{N}}{\partial x}(1, t)+\alpha\left(u_{N}(1, t)-g(t)\right)=0 .
$$

Stability and spectral accuracy is obtained if the coefficient $\alpha$ is chosen of the order $N^{2}$.

This technique was later called SAT methods from Simultaneous Approximation Term. It has become quite popular, and is now used as a standard technique also for difference methods. For internal boundaries arising from material discontinuities or domain decomposition methods, it is particularly convenient.

\section{Gibbs phenomenon}

When approximating a discontinuous function by a finite Fourier series, nonphysical oscillations occur. No matter how many terms are used, there will always be a point next to the discontinuity that has an $\mathcal{O}(1)$ error as shown in Fig. 5. This type of oscillations will occur whenever spectral methods are used for PDE, if the solution has shocks or 


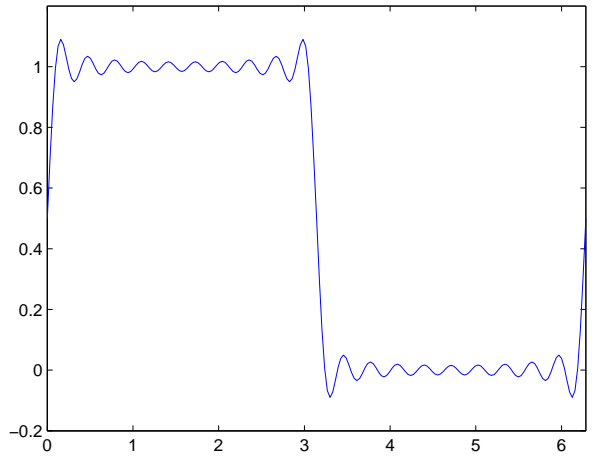

(a) $\mathrm{N}=40$

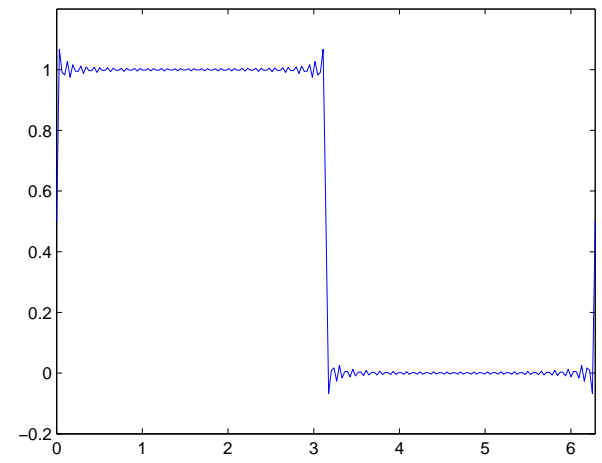

(b) $\mathrm{N}=160$

Figure 5: Fourier series approximation of a discontinuous function.

contact discontinuities. Because of the global character of polynomial methods, they will show the same type of behavior as Fourier methods do. This is a serious limitation of spectral methods, but David and others set out to do something about it.

The first paper on this topic was [41], where an improvement of the original oscillatory solution was obtained by convolution with a regularization kernel, see also [5]. In [9] even better solutions were obtained by adding a sawtooth function to the original Fourier modes. The improvement here was the removal of the error also in the immediate neighborhood of the discontinuity.

Ami Harten was at the time one of the leading researchers in shock capturing finite difference methods, and a collaboration with him and David's former student Wei Cai produced an essentially nonoscillating Chebyshev method, [8]. The central problem here is the transformation between point values and cell averages. This is a trivial operation for finite difference and Fourier methods, but not for Chebyshev polynomials.

The paper [10] started a new approach by considering the basic approximation problem of representing a discontinuous function by a finite spectral expansion. By using a least square procedure for constructing one-sided filters, the Gibbs phenomenon was removed. This was followed up in a fruitful cooperation with Chi-Wang Shu, which resulted in no less than 5 papers called "On the Gibbs Phenomenon I,II,III,IV,V", see [3640]. The main approach is the introduction of Gegenbauer polynomials, which satisfy

$$
\int_{-1}^{1}\left(1-x^{2}\right)^{\lambda-\frac{1}{2}} \phi_{j}^{\lambda}(x) \phi_{k}^{\lambda}(x) d x=0, \quad j \neq k,
$$

where $\lambda$ is a free parameter. The Fourier series, which converges poorly, is postprocessed by expanding it in a Gegenbauer series. The authors prove that this results in an exponentially accurate solution in any subinterval where the solution is analytic.

The Galerkin case is treated in the first four papers, while the collocation case is treated in the fifth one. The whole series of papers is pure approximation theory without 


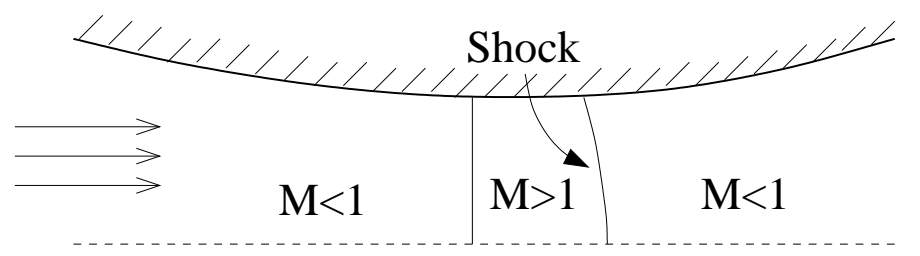

Figure 6: Nozzle flow.

any connection to differential equations. The basic question is how to get a good approximation of a given discontinuous function, with known location of the discontinuity. When solving PDE with spectral methods, the location is part of the solution, and the first problem is to find it. Various methods have been used for this including edge detection techniques applied by David's former student Anne Gelb and others. The situation today is quite satisfactory for problems in one space dimension, but in several dimensions we are not yet there.

The difficulties with discontinuities are not limited to numerical solutions obtained by spectral methods, they show up with all shock capturing methods. Many well working difference methods based on some form of upwinding have been developed during the last decades. However, there is one remaining difficulty. If one family of characteristics is propagating through the shock, the accuracy deteriorates on the downstream side regardless of the formal high order accuracy of the basic method. The nozzle problem with flow governed by the Euler equations is such an example. Subsonic flow is entering from the left, goes supersonic at the throat, and goes subsonic again across a shock, see Fig. 6. Here David came up with a new idea together with his daughter Sigal Gottlieb and Chi-Wang Shu. The steady state nozzle problem was solved in [47] by using a high order WENO difference scheme. In order to recover the high accuracy downstream, the grid values of the numerical solution are used as the basis for Fourier interpolation. This Fourier series is then used for Gegenbauer reconstruction as described above, and the numerical results are very good.

This is a nice example of the influence of David's work. The work on Gibbs phenomenon was initiated by the difficulties with spectral methods. However, the results did not only influence the use of spectral methods, but also helped solving the well known difficulty with accuracy deterioration for shock capturing difference methods.

\section{High order difference methods}

When David came to MIT, he had just completed his PhD thesis. It was about so called Strang type difference methods

$$
\mathbf{u}^{n+1}=L_{1}\left(\mathbf{u}^{n}\right) L_{2}\left(\mathbf{u}^{n}\right) \cdots L_{d}\left(\mathbf{u}^{n}\right)
$$


for nonlinear conservation laws

$$
\frac{\partial \mathbf{u}}{\partial t}=\sum_{j=1}^{d} \frac{\partial \mathbf{F}_{j}(\mathbf{u})}{\partial x_{j}}
$$

and he came to the right place since Gilbert Strang was a real specialist on this type of methods. In [21] there is a thorough analysis of new schemes regarding accuracy, stability and efficacy.

He followed up with a series of papers on stability and other key properties of various schemes, in most cases for multidimensional problems, see $[1,6,15,44,45,54]$. Let us take a look at the last one treating the conservation law

$$
\frac{\partial \mathbf{u}}{\partial t}=\frac{\partial \mathbf{f}(\mathbf{u})}{\partial x}+\frac{\partial \mathbf{g}(\mathbf{u})}{\partial y} .
$$

The method of lines was at the time (and still is) very popular. It means that a discretization is first done in space, followed up by an application of an ODE-solver to the resulting system of ordinary differential equations. Explicit Runge-Kutta methods are often chosen for the ODE-solver. However, there is one complication here. Fourth order accuracy in space results in a 5-point stencil for first order PDE, but for each stage in the Runge-Kutta procedure, the width increases by 4 points. A fourth order method with four stages results in a 17 point stencil in each space dimension. When boundaries are involved, the analysis becomes complicated. More compact schemes are obtained by using the Lax-Wendroff principle, and David and his coworkers picked up this idea, but in a more advanced form. Their final scheme has four stages in each step just like the standard Runge-Kutta scheme, but by keeping each stage as compact as possible, the complete computational stencil reduces from 289 to 16 points in two space dimensions, see Fig. 7. In three space dimensions the reduction is of course even stronger.

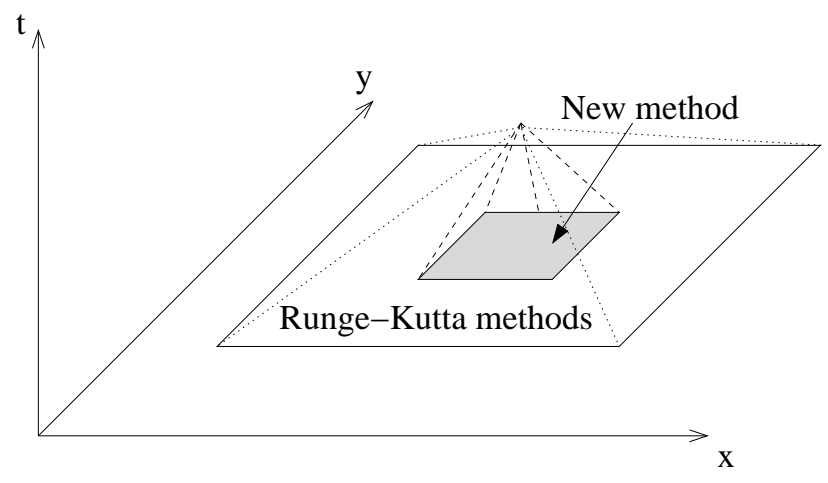

Figure 7: Reduction of computational stencil size.

The stability analysis for the linear case is not easy for this scheme, but the authors manage to derive the explicit form of the amplification factor in Fourier space after "con- 
siderable amount of manipulation". The final stability criterion has the simple form

$$
\frac{\Delta t}{\Delta x} \rho\left(\mathbf{f}^{\prime}\right) \leq 1, \quad \frac{\Delta t}{\Delta y} \rho\left(\mathbf{g}^{\prime}\right) \leq 1,
$$

where $\rho\left(\mathbf{f}^{\prime}\right)$ and $\rho\left(\mathbf{g}^{\prime}\right)$ denote the Jacobians.

Difference methods dominated the first part of David's production, and reoccurred a few times later as well. He was much interested in the so called Summation By Parts (SBP) difference operators that lead to energy estimates (for a general description of these operators, see for example [48]). In [27] the connection between compact finite difference operators and finite element methods is used to analyze the problem of constructing high order numerical boundary conditions for hyperbolic problems. In [11] the SAT technique mentioned in Section 3 is introduced for difference approximations of advection-diffusion problems. The computational domain is partitioned into subdomains, and continuity is enforced by using the SAT method for the internal boundaries.

\section{Open boundaries and the PML method}

Many problems are defined over infinite domains, and for computational purposes, artificial boundaries must be introduced as in the example shown in Fig. 8. Many attempts to solve the problem of constructing boundary conditions at these boundaries have been made, and there is a wide variety of different techniques. Berenger presented the Perfectly Matched Layer (PML) method for problems in electromagnetics 1994 in [7], and this has come to be one of the most frequently used methods. The idea is to extend the computational domain by an extra layer, where the system of differential equations is modified such that the waves are absorbed. The technique was designed for electromagnetics, but it was quickly generalized to other applications. However, a few less well behaving computations with this method were observed, and together with Saul Abarbanel, David set out to analyze the new modified differential equations without applying any discretization. In the first paper [2] they showed that the modified PDE for electromagnetics, to be used in the extra layer, is not strongly well posed, i.e., the symbol has a multiple eigenvalue that causes trouble. It was followed up by another paper [3], where they construct a new modified set of PDE with an extra equation, where the source of trouble is removed.

However, the problem was still not completely solved. Even for the new method, some computations showed that after a very long time, the solution began growing in a way that destroyed the absorbing property. In [4] this behavior was analyzed, and again there is a double eigenvalue even for the new equations, but now originating from the zero order term. The suggested cure is to split these eigenvalues into two separated ones by introducing a small parameter $\varepsilon$. However, as the authors point out, there is a balancing problem here. The parameter $\varepsilon$ must be kept small to keep the absorption of waves complete, while at the same time it must be large enough to keep the solution bounded. 


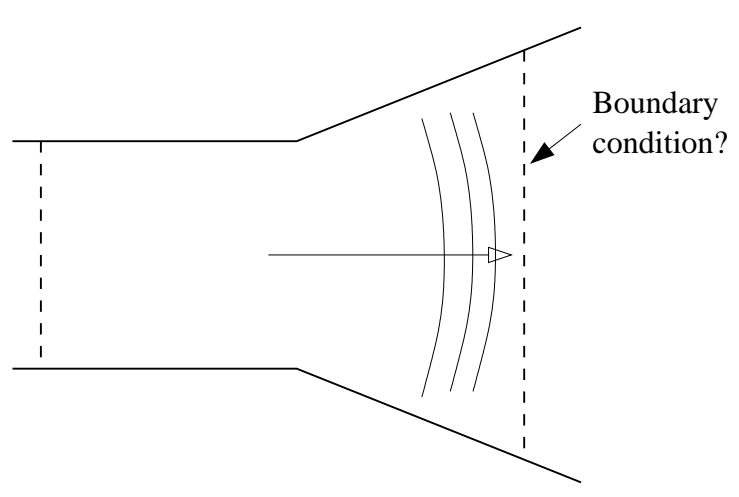

Figure 8: A problem requiring an artificial boundary.

David had also an interesting article [14] together with Adi Ditkowski on the classical Engquist-Majda absorbing boundary conditions. They derived the same type of boundary conditions for the wave equation by using a different technique. But this technique is more general, and it allowed them to derive the corresponding type of conditions to the three-dimensional Maxwell equations as well as for the advection-diffusion equation.

\section{Other areas}

David was active in many different research areas, and in this section we shall very briefly mention some of his work that has not been described above.

Spectral polynomial methods have a disadvantage in the sense that the distribution of grid points is determined by the polynomials themselves, not by the structure of the solution. For example, the Chebyshev polynomials concentrate the points near the boundaries, whether or not the solution has any boundary layers. This makes it natural to partition the domain into subdomains with different polynomials defined in each one of them. In this way the smallest grid size can be kept above a certain reasonable level. This may have been a reason for David's interest in domain decomposition methods, see $[13,16,28,29,46]$.

In the nineties, David started working with Roger Temam on nonlinear Galerkin methods. It was natural for them to use spectral methods for this new formulation, and some of the results are presented in [12,43].

A different problem, not connected to PDE at all, was presented to David by this author. Most methods for computer tomography are based on Fourier transforms, and the speed is essential. In [26] a variant of the so called Direct Fourier Method is presented, with a new type of interpolation in Fourier space, that improves the image quality without slowing down the computation too much.

During the last years, he also went into stochastic problems, discontinuous Galerkin methods and particle methods.

Finally we would like to point out David's strong interest in various applications. 


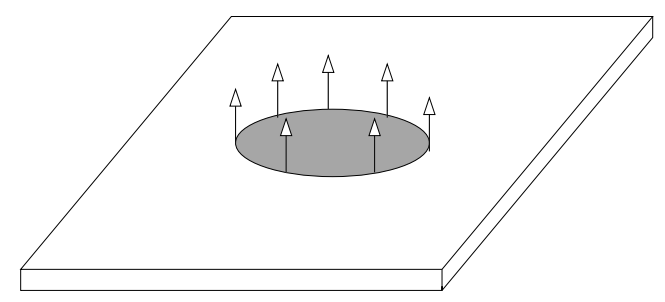

Figure 9: The von Karman plate.

Due to his connection to NASA Langley Research Center, fluid dynamics was the most common application in his work, but he got involved in many other applications as well. We mention one such example here. The application by others of Chebyshev methods to the von Karman plate problem had not turned out well. The problem is to compute the displacements $u, v, w$ when a force is applied to the center as shown in Fig. 9.

The first differential equation in the system is

$$
\begin{aligned}
w_{t t} & +c w_{t}+\Delta^{2} w-12\left(\left(u_{x}+\frac{1}{2} w_{x}^{2}\right)\left(w_{x x}+v w_{y y}\right)\right. \\
& \left.+\left(v_{y}+\frac{1}{2} w_{y}^{2}\right)\left(w_{y y}+v w_{x x}\right)+(1-v)\left(v_{x}+u_{y}+w_{x} w_{y}\right) w_{x y}\right)=g .
\end{aligned}
$$

The basic difficulty with the computations was the strong filtering that seemed to be necessary in order to keep the computation stable. There was a general belief that the nonlinear terms in the differential equation were the cause of the problem. However, David became involved, and a stability analysis showed that there was an instability even for the linearized system due to the mixed derivative terms. Furthermore, this instability could be removed by switching to Legendre polynomials. There was still a nonlinear instability, but it could be taken care of by a different and weaker form of filter as presented in [50]. It is a nice example out of many, where David helped to advance the understanding of a difficult problem in applications, and to speed up its solution.

\section{Conclusions}

David Gottlieb's research career is a success story, in particular the work on spectral methods, as well as the related Gibbs phenomenon. He took the idea of spectral methods to new levels of refinement, and was the first one to carry out a theoretical analysis of polynomial methods. He was the one who introduced spectral methods to the European scientific community by giving a course in France 1979. This resulted in a fast expanding interest from several young researchers in France and Italy. Christine Bernardi, Yvon Maday, Claudio Canuto and Alfio Quarteroni were among the pioneers who carried the theory further, resulting in new variants of spectral methods as well as application to new problems.

What was David's special properties that made him such an excellent researcher and scholar? It goes without saying that he was very sharp and creative. But there are also 
other ingredients. This author was often impressed by his deep and broad knowledge in classical and applied mathematics, that turned out to be such an important tool in his work. Not to talk about his skill in calculus. He was able to quickly find integrals of some complicated function, or alternatively, to immediately find the place in some book where to find it. And he had an unusual talent in seeing patterns that untied a complicated mathematical expression or equation.

David was always curious about other's work and other research areas. And he was open-minded and generous. Whoever came with a new problem, student, colleague or representative for a high tech laboratory, he always took a genuine interest, sharing his ideas with others. It is hard to find mathematicians on his level with so many collaborators. His 125 papers have 59 different coauthors, which is impressive when taking into account that there are few papers with more than one coauthor.

His teaching abilities are well known, as well as his guidance of graduate students. He led $22 \mathrm{PhD}$ students to completed degree.

David Gottlieb was an extraordinary man. This article is completed right after the conference in honor of him took place. There was no doubt that all the nice remarks about him were genuine and came from the heart of the speakers. Not only was he an excellent researcher and scholar, he was also an extremely kind and generous person. We would like to quote Antony Jameson at the conference banquet: "I don't think that David ever thought a mean thought".

\section{References}

[1] S. Abarbanel and D. Gottlieb, High order accuracy finite difference algorithm for quasilinear conservation law hyperbolic systems, Math. Comput., 27 (1973), 505-523.

[2] S. Abarbanel and D. Gottlieb, A mathematical analysis of PML methods, J. Comput. Phys., 134 (1997), 357-363.

[3] S. Abarbanel and D. Gottlieb, On the construction and analysis of absorbing layers in CEM, Appl. Numer. Math., 27 (1998), 331-340.

[4] S. Abarbanel, D. Gottlieb and J. Hesthaven, Long time behaviour of the perfectly matched layer equations in computational electromagnetics, J. Sci. Comput., 17 (2002), 405-422.

[5] S. Abarbanel, D. Gottlieb and E. Tadmor, Spectral methods for discontinuous problems, Numerical Methods for Fluid Dynamics II (Ed: Morton and Baines), Oxford University Press, 1986, 128-153.

[6] S. Abarbanel, D. Gottlieb and E. Turkel, Difference schemes with fourth order accuaracy for hyperbolic equations I, SIAM J. Appl. Math., 13 (1975), 329-351.

[7] J.-P. Berenger, A perfectly matched layer for the absorbtion of electromagnetic wave, J. Comput. Phys., 114 (1994), 185-200.

[8] W. Cai, D. Gottlieb and A. Harten, Cell averages Chebyshev methods for hyperbolic problems, Comput. Math. Appl., 24 (1992), 37-49.

[9] W. Cai, D. Gottlieb and C. W. Shu, Essentially non-oscillatory spectral Fourier methods for shock wave calculations, Math. Comput., 52 (1989), 389-410.

[10] W. Cai, C. W. Shu and D. Gottlieb, On one sided filters for spectral Fourier approximations of discontinuous functions, SIAM J. Numer. Anal., 29 (1992), 905-916. 
[11] M. Carpenter, D. Gottlieb and J. Nordström, A stable and conservative interface treatment of arbitrary spatial accuracy, J. Comput. Phys., 148 (1999), 341-365.

[12] L. Dettori, D. Gottlieb and R. Temam, A nonlinear Galerkin method: the two level Fourier collocation case, J. Sci. Comput., 10 (1995), 371-389.

[13] W. S. Don, D. Gottlieb and J. H. Jung, A multi-domain method for supersonic reactive flows, J. Comput. Phys., 192 (2003), 325-354.

[14] A. Ditkowski and D. Gottlieb, On the Engquist Majda absorbing boundary conditions for hyperbolic equations, Contemp. Math., 330 (2003), 55-71.

[15] B. Eilon, D. Gottlieb and G. Zwas, Numerical stabilizers and computing time for second order accurate schemes, J. Comput. Phys., 9 (1972), 387-397.

[16] P. Fischer and D. Gottlieb, On the optimal number of subdomains for hyperbolic problems on parallel computers, Int. J. Supercomput. Ap., 11 (1997), 65-76.

[17] B. Fornberg, On high order approximations of hyperbolic differential equations by a Fourier method, Report No. 39, Dept. Comput. Science, Uppsala University, 1972.

[18] D. Funaro, Domain decomposition for pseudo spectral approximations-part one: second order equations in one dimension, Numer. Math., 52 (1988), 329-344.

[19] D. Funaro and D. Gottlieb, A new method of imposing boundary conditions in pseudospectral approximations of hyperbolic equations, Math. Comput., 51, (1988), 599-613.

[20] D. Funaro and D. Gottlieb, Convergence results for pseudospectral approximations of hyperbolic systems by a penalty type boundary treatment, Math. Comput., 57, (1991), 585-596.

[21] D. Gottlieb, Strang type diference schemes for multi-demesional problem, SIAM J. Numer. Anal., 9 (1972), 650-661.

[22] D. Gottlieb, On the stability of Rusanov's third order scheme, J. Comput. Phys., 15 (1974), 421-426.

[23] D. Gottlieb, The stability of pseudospectral-Chebyshev, Math. Comput., 36 (1981), 107-118.

[24] D. Gottlieb and P. Fischer, On the optimal number of subdomains for hyperbolic problems on parallel computers, 11 (1997), 65-76.

[25] D. Gottlieb and B. Gustafsson, Generalized Du-Fort Frankel methods for parabolic initial boundary value problems, SIAM J. Appl. Math., 29 (1976), 329-351.

[26] D. Gottlieb, B. Gustafsson and P. Forsse'n, On the direct Fourier method for computer tomography, IEEE Trans. Medic. Imag., 19 (2000), 223-232.

[27] D. Gottlieb, B. Gustafsson, P. Olsson and B. Strand, On the superconvergence of Galerkin methods for hyperbolic IBVP, SIAM J. Numer. Anal., 33 (1996), 280-290.

[28] D. Gottlieb and R. Hirsh, Parallel pseudospectral domain decomposition techniques, J. Sci. Comput., 4 (1989), 309-325.

[29] D. Gottlieb and M. S. Min, Domain decomposition spectral approximations for an eigenvalue problem with a piecewise constant coefficient, SIAM J. Numer. Anal., 43 (2005), 502520.

[30] D. Gottlieb and C. E. Wasberg, Optimal decomposition of the domain in spectral methods for wave like phenomenon, SIAM J. Sci. Comput., 22 (2000), 617-632.

[31] D. Gottlieb and L. Lustman, The Du-Fort Frankel Chebyshev method for parabolic initialboundary value problems, Comput. Fluids., 11 (1983), 107-120.

[32] D. Gottlieb, L. Lustman and E. Tadmor, Stability analysis of spectral methods for hyperbolic initial boundary value problems, SIAM J. Numer. Anal., 24 (1987), 241-256.

[33] D. Gottlieb, L. Lustman and E. Tadmor, Convergence of spectral methods for hyperbolic initial boundary value problems, SIAM J. Numer. Anal., 24 (1987), 532-537.

[34] D. Gottlieb and S. Orszag, Numerical Analysis of Spectral Methods: Theory and Applica- 
tions, CBMS-NSF 26, Philadalphia: SIAM, 1977.

[35] D. Gottlieb, S. Orszag and E. Turkel, Stability of pseudospectral and finite difference methods for variable coefficient problems, Math. Comput., 37 (1981), 293-305.

[36] D. Gottlieb, C.-W. Shu, A. Solomonoff and H. Vandeven, On the Gibbs phenomenon I: recovering exponential accuracy from the Fourier partial sum of a non-periodic analytic function using Gegenbauer polynomials, J. Comput. Appl. Math., 43 (1992), 81-98.

[37] D. Gottlieb and C.-W. Shu, On the Gibbs phenomenon II: resolution properties of the Fourier method for discontinuous waves, Comput. Meth. Appl. Mech. Eng., 116 (1994), 27-37.

[38] D. Gottlieb and C.-W. Shu, On the Gibbs phenomenon III: recovering exponential accuracy in a sub-interval from a spectral partial sum of a piecewise analytic function using Gegenbauer polynomials, SIAM J. Numer. Anal., 33 (1996), 280-290.

[39] D. Gottlieb and C.-W. Shu, On the Gibbs phenomenon IV: recovering exponential accuracy in a sub-interval from a Gegenbauer partial sum of a piecewise analytic function, Math. Comput., 64 (1995), 1081-1095.

[40] D. Gottlieb and C.-W. Shu, On the Gibbs phenomenon V: recovering exponential accuracy from collocation point values of a piecewise analytic function, Numer. Math., 71 (1995), 511526.

[41] D. Gottlieb and E. Tadmor, Recovering pointwise value of discontinuous data within spectral accuracy, U.S.-Israel Workshop, Progress in Scientific Computing (Ed: Murman, Abarbanel), Birkhauser Boston, 1985, 357-376.

[42] D. Gottlieb and E. Tadmor, The CFL condition for spectral approximations to hyperbolic initial-boundary value problems, Math. Comput., 56 (1991), 565-588.

[43] D. Gottlieb and R. Temam, Implementation of the nonlinear Galerkin method with pseudospectral (collocation) discretizations, Appl. Numer. Math., 12 (1993), 119-134.

[44] D. Gottlieb and E. Turkel, Phase error and stability of second order methods for hyperbolic problems, J. Comput. Phys.,15 (1974), 251-265.

[45] D. Gottlieb and E. Turkel, Dissipative Two-Four methods for time dependent problems, Math. Comput., 136 (1976), 703-723.

[46] D. Gottlieb and C. E. Wasberg, Optimal decomposition of the domain in spectral methods for wave like phenomenon, SIAM J. Sci. Comput., 22 (2000), 617-632.

[47] S. Gottlieb, D. Gottlieb and C.-W. Shu, Recovering high order accuracy in WENO computations of steady state hyperbolic systems, J. Sci. Comput., 28 (2006), 307-318.

[48] B. Gustafsson, High Order Difference Methods for Time Dependent PDE, Springer Series in Computational Mathematics, Springer, 2008.

[49] J. Hesthaven, S. Gottlieb and D. Gottlieb, Spectral Methods for Time-Dependent Problems, Cambridge University Press, 2006.

[50] M. Kirby, Z. Yoosibash and D. Gottlieb, Collocation methods for the solution of the von Karman dynamical nonlinear plate systems, J. Comput. Phys., 200 (2004), 432-461.

[51] H.-O. Kreiss and J. Oliger, Comparison of accurate methods for the integration of hyperbolic equations, Tellus., 24 (1972), 199-215.

[52] H.-O. Kreiss and J. Oliger, Methods for the Approximate Solution of Time Dependent Problems, GARP Publication Series, No. 10, 1973.

[53] S. Orszag, Numerical simulation of incompressible flows within simple boundaries, I, Stud. Appl. Math., 50 (1971), 293-327.

[54] E. Turkel, S. Abarbanel and D. Gottlieb, Multidimensional difference schemes with fourth order accuracy, J. Comput. Phys., 21 (1976), 85-113. 